\title{
Salmon On The Contingent A Priori And The Necessary A Posteriori
}

In Naming And Necessity ${ }^{1}$, Saul Kripke suggests that there may be contingent propositions which are knowable a priori (by creatures like us), and that there may be necessary propositions which are knowable only a posteriori (by creatures like us). As an example of a contingent proposition which is knowable a priori (by creatures like us) Kripke offers: the proposition which is the content of the sentence The length of stick $S$ at $t_{o}$ is one metre, where stick $S$ is the standard metre, and $t_{0}$ is the time at which the reference of the expression "one metre" is fixed for one via reference to (the length of) S. And, as an example of a necessary proposition which is knowable only a posteriori (by creatures like us) Kripke suggests: the proposition which is the content of the sentence Hesperus is Phosphorus.

In Frege's Puzzle ${ }^{2}$, Nathan Salmon argues that, although the general claims which Kripke makes are correct, the examples which he gives do not carry conviction. On the one hand, Salmon argues that, although there are necessary propositions which are knowable only a posteriori (by creatures like us), the sentence Hesperus is Phosphorus actually has as its content a necessary proposition which is knowable $a$ priori (by creatures like us). And, on the other hand, Salmon argues that although there are contingent propositions which are knowable a priori (by creatures like us), the sentence The length of stick $S$ at to is one metre actually has as its content a contingent proposition which is only knowable a posteriori (by creatures like us).

In this paper, I propose to argue: (i) that if Salmon's case against Kripke's examples is good, then it can be extended to show that there are no examples of the contingent $a$ priori; and (ii) that if Salmon were to grant that Kripke's examples are good, while maintaining the rest of his views, then he would very likely be committed to the conclusion that all singular propositions -- or, at least, all suitably existentially 
circumscribed singular propositions -- can be known a priori. ${ }^{3}$ I shall conclude with some suggestions about how Salmon's views ought to be amended.

In order to assess Salmon's arguments, it will be necessary to keep a large part of his theory in view. Since his theory is quite complex, the following overview is only intended to pick out the most important features for present purposes.

1. Salmon draws a sharp distinction between, on the one hand, logical attributes -e.g. logical validity, logical truth, consistency, entailment -- which apply "primarily, and in the first instance" to sentences, or sets of sentences; and, on the other hand, epistemological and metaphysical attributes -- e.g. a priority, a posteriority, informativeness, necessity, contingency -- which apply "primarily, and in the first instance" to propositions (pieces of cognitive information). ${ }^{4}$ Although Salmon makes no explicit pronouncement about this, I believe that he holds that truth is an attribute which applies "primarily, and in the first instance" to propositions. However, since Salmon seems to allow that there are some attributes -- e.g. analyticity -- which do not have clear primary application to either sentences or propositions, it may be that truth also belongs in this mixed category. ${ }^{5}$

2. Salmon holds that the semantic contents of tokenings of sentences (with respect to contexts of tokening) are structured propositions; moreover, he holds that the semantic contents of tokenings of many sentences are singular propositions -- i.e. propositions which have particular objects as constituents. In particular, the semantic contents of tokenings of sentences which contain occurrences of proper names, 
indexicals, and demonstratives -- not embedded in quotational or other special linguistic constructions -- are singular propositions.

3. Salmon holds that propositional attitudes should be analysed as mediated binary relations between subjects and structured propositions, where the mediating entities are ways of taking those propositions. Thus, for example, he holds that belief should be analysed as one kind of binary relation which holds between subjects and structured propositions, mediated by ways of taking those propositions. Salmon does not provide a positive characterisation of "ways of taking". He does say that ways of taking are neither "Fregean senses", nor sentences in natural language, nor sentences in neural language (language of thought). Moreover, he allows that information about ways of taking can be conveyed via the utterance of sentences in natural language.

4. Salmon holds that ways of taking propositions do not figure in the truth-conditions for propositional attitude ascriptions. Thus, for example, he holds that a suitable generalisation of the following principle provides the truth-conditions for belief ascriptions:

(S) A believes that $p$ is true iff $(\exists x)(x$ is a way of taking the structured proposition that $\mathrm{p}$, and $\mathrm{A}$ grasps that structured proposition that $\mathrm{p}$ by means of $\mathrm{x}$, and BEL (A, the structured proposition that $\mathrm{p}, \mathrm{x}$ ))

5. Notoriously, Salmon's account of the truth-conditions for belief ascriptions has the consequence that directly referential co-referring singular terms (e.g. proper names, indexicals, demonstratives) are intersubstitutable salva veritate. However, Salmon holds that tokenings of belief ascriptions also carry conventional (or generalised) Gricean implicatures about the ways of taking which are involved in the attributed beliefs. Moreover, Salmon contends that it is a confusion, of this pragmatically imparted information with the properly semantic information which is encoded in 
belief reports, which generates those intuitions which suggest that directly referential co-referring singular terms are not intersubstitutable in belief reports salva veritate.

6. Salmon explicitly notes that it is "a large and difficult problem" to specify what kinds of connections between subjects and objects can suffice to establish that subjects have ways of taking singular propositions. What he says is that a "de re" connection is required; what is unclear is just which connections are "de re". ${ }^{6}$ More generally, there is a large open question in Salmon's work about the connections between (i) ways of taking objects; (ii) conditions under which a subject has de re thoughts about objects; and (iii) conditions under which a subject knows which object is the object currently under consideration.

7. There is an obvious question which arises for Salmon's theory concerning empty singular terms. What, e.g., is the proposition -- if any! -- which is the content of the sentence "Santa Claus has a white beard"? Different answers to this question are suggested by different parts of Salmon's work.

(i) In Frege's Puzzle, Salmon suggests -- to me at least -- that he is tempted by the view that this sentence expresses no proposition of any sort. ${ }^{7}$ On this view, the sentence "Jenny believes that Santa Claus has a white beard" also expresses no proposition of any sort. Nonetheless, this latter sentence could be taken to pragmatically impart information about Jenny -- viz. that she has a certain "way of taking" (even though there is no proposition with which that way of taking makes contact).

(ii) In a later work, Salmon defends the view that (many) empty singular terms directly refer to merely possible objects, and that these objects can be the constituents of merely possible propositions. ${ }^{8}$ On this view -- and granted the assumption that "Santa Claus" is an empty singular term -- the sentence "Jenny believes that Santa 
Claus has a white beard" expresses a proposition which has, as one of its constituents, a proposition which does not exist, but which might have existed. (This proposition, in turn, has as one of its constituents, Santa Claus -- i.e. a being which does not exist, but which might have existed, and to which the term "Santa Claus" directly refers.)

(iii) In correspondence, Salmon has informed me that he holds that "Santa Claus" refers to a fictional or mythological character -- i.e. that it is not a genuinely nonreferring term. Moreover, he suggests that it is important to distinguish among the following cases of "empty" singular terms: (a) names from fiction; (b) names from myth; (c) names for past individuals which no longer exist; (iv) names for future individuals which do not yet exist; (v) names for forever merely possible individuals; (vi) names for (certain) impossible individuals. Finally, he notes that there probably are genuinely non-referring terms, but that it is difficult to provide an example. ${ }^{9}$

Salmon's argument that Kripke provides an incorrect assessment of the status of the proposition expressed by the sentence Hesperus is Phosphorus is straightforward. On Salmon's view -- a view which Kripke himself does not necessarily reject -- the proposition expressed by the sentence Hesperus is Phosphorus is a singular proposition which contains two occurrences of the planet Venus. This proposition is clearly necessary. Moreover, on Salmon's view, it is also clearly knowable a priori: anyone who has a way of taking the planet Venus can grasp this necessary proposition a priori via the way of taking whose constituents include two occurrences of the very same way of taking the planet Venus. 
Given Salmon's account of propositional attitude ascriptions, it follows that, strictly speaking, it is knowable a priori that Hesperus is Phosphorus. However, it does not follow from this that the sentence It is knowable a priori that Hesperus is Phosphorus is correctly assertable. Indeed, it would seem that, on Salmon's view, that sentence is not correctly assertable: for it carries the false implicature that the proposition which is the content of the sentence Hesperus is Phosphorus can be known a priori when it is grasped via the (or a) way of taking which is naturally associated with that sentence. Rather, it would seem that the relevant sentence which is correctly assertable is this: Strictly speaking, it is knowable a priori that Hesperus is Phosphorus.

There are two different ways in which Salmon might be taken to be urging a revision of our practice of making propositional attitude ascriptions. On the first, and more radical, alternative, Salmon would be taken to be urging a broad-ranging revision according to which, e.g., it will be correct to assert the sentence Lois Lane knows ( $a$ priori) that Superman is Clark Kent, and false to assert the sentence Lois Lane does not know (even a posteriori) that Superman is Clark Kent. On this alternative, Salmon would be taken to hold that there is no sense in which ordinary folk correctly say that one can only know a posteriori that Hesperus is Phosphorus. Rather, in any circumstances, the only correctly assertable claim is that one can know a priori that Hesperus is Phosphorus.

On the second, and more conservative, alternative, Salmon would be taken to allow only that, in contexts in which we are speaking strictly, we ought, e.g., to assent to the sentence Lois Lane knows (a priori) that Superman is Clark Kent, and not to assert to the sentence Lois Lane does not know (even a posteriori) that Superman is Clark Kent. That is, the suggestion is not that we ought to revise our practice tout court; and so, on this alternative, Salmon would be taken to allow that, speaking with the ordinary folk, one can only know a posteriori that Hesperus is Phosphorus. 
However, he would also be taken to allow that, speaking strictly, one can know $a$ priori that Hesperus is Phosphorus..$^{10}$ And consequently, given that Kripke intended to speak with full philosophical seriousness, it would seem to follow -- as it did on the first alternative -- that Kripke was wrong about the status of the claim that Hesperus is Phosphorus. ${ }^{11}$

In my view, there is good reason for Salmon to adopt the more conservative alternative. In particular, it is obvious that often we want information about the ways in which propositions are taken -- and moreover it seems clear that the language which we currently speak makes provision for effective communication of such information. Also, I think that Salmon would agree that it would be very cumbersome to speak a language in which explicit reference to ways of taking is always required -i.e. to speak a language in which ways of taking always feature as constituents of the propositions which are the semantic contents of sentences, and never as constituents of the propositions which are the pragmatic contents of sentences. So it seems to me that Salmon ought not want to insist on a complete revision of our practice.

However, in correspondence, Salmon has (I think) claimed that he defends the more radical alternative. Certainly, he says that the sentence "It is knowable a priori that Hesperus is Phosphorus" is assertable (in the only sense in which he would use that term), even though it is misleading and inappropriate; and he also says that there is no sense in which the sentence "It is knowable only a posteriori that Hesperus is Phosphorus" is assertable. Unless this is just a trivial dispute about the meaning of the words "correctly assertable" -- one which arises because Salmon has stipulatively assigned them an unorthodox meaning -- it seems to me that Salmon is thus committed to the radical conclusion that there is no sense in which our ordinary practice of making and using propositional attitude ascriptions is in order as it stands, and that it ought to be revised. ${ }^{12}$ 
III

Salmon's argument that Kripke does not provide a correct assessment of the status of the proposition which is the content of the sentence The length of stick $S$ at $t_{o}$ is one metre is also apparently straightforward. ${ }^{13}$

Suppose, for the sake of argument, that the sentence The length of stick $S$ at $t_{o}$ is one metre has as its semantic content the Russellian proposition < the-length-of-stick-Sat- $t_{0}$, being-the-length-of-stick-S-at- $t_{0}>$. Here, we suppose that the-length-of-stick-Sat- $t_{0}$ is an abstract quality -- i.e. a species of the generic Lockean primary quality length -- and that being-the-length-of-stick-S-at- $\mathrm{t}_{\mathrm{o}}$ is a complex property. No doubt the propositional content of the sentence in question is not really this simple; however, Salmon supposes that any further complexity will be irrelevant to his argument. $^{14}$

Given the theory to which Salmon subscribes, it follows that one who uses the expression the length of $S$ at $t_{o}$ to fix the reference of the expression one metre knows a priori that the length of stick $\mathrm{S}$ at $\mathrm{t}_{\mathrm{o}}$ is one metre iff that person knows the Russellian proposition < the-length-of-stick-S-at- $\mathrm{t}_{\mathrm{o}}$, being-the-length-of-stick-S-at- $\mathrm{t}_{\mathrm{o}}$ $>$ a priori . But, Salmon claims, the reference-fixer knows the Russellian proposition $<$ the-length-of-stick-S-at- $\mathrm{t}_{0}$, being-the-length-of-stick-S-at- $\mathrm{t}_{\mathrm{o}}>$ a priori only if s/he knows without appeal to experience of the-length-of-S-at- $\mathrm{t}_{0}$ that it has precisely that length. "Yet it would seem that no matter what stipulation one makes, one cannot know without resorting to experience such things as that $\mathrm{S}$.. has precisely such-andsuch particular length at $t_{0}$. It would seem that one must at least look at $S$ 's length, or be told that it is precisely that long, etc. Therefore, it would seem that the metre sentence is not a priori but a posteriori.”. ${ }^{15}$ 
It is tempting to respond to this argument as follows: The reference-fixer takes one metre to be the length of stick $S$ at $t_{0}--$ i.e. it is part of the way in which the reference-fixer takes the length one metre that s/he takes it to be the length of stick S at $\mathrm{t}_{0} \cdot{ }^{16}$ Consequently, the reference-fixer need only reflect on the way in which $\mathrm{s} / \mathrm{he}$ takes the length one metre in order to come to know that one metre is the length of stick $\mathrm{S}$ at $\mathrm{t}_{\mathrm{o}}$. So experience need play no part in the justification of the referencefixer's belief. Of course, experience has a role in the reference-fixer's acquisition of the way in which s/he takes the length one metre -- but, once given the acquisition of that way of taking the length one metre, experience plays no further role in the move to the belief (and indeed knowledge) of the proposition that one metre is the length of stick $S$ at $t_{0}$.

The distinction to which I have adverted, between two different roles which experience might play in ways of taking propositions, is introduced by Salmon himself. He writes: "A piece of knowledge is a priori if sensory experience need not play a certain key role in its justification. ... This is what I claim for the singular proposition about Venus that it is it. ... One can know the proposition on the basis of reflection .. alone by taking it the way one would if one stipulated that one is considering a certain trivial truism -- as in 'Consider the fact about Venus that it is it.' That fact is thus knowable without recourse to sensory experience.” ${ }^{17}$ But the point is that it seems that one can equally insist that one can know the singular proposition about the length one metre -- that it is the length of stick $\mathrm{S}$ at $\mathrm{t}_{\mathrm{o}}$-- by reflection alone, if one takes it in the way one would if one stipulated that one was considering a certain trivial truism. Given that I use the description "the length of stick S at $\mathrm{t}_{\mathrm{o}}$ " to fix the reference of the expression one metre", it is a trivial truism that "The length of stick $\mathrm{S}$ at $\mathrm{t}_{\mathrm{o}}$ is one metre". This case seems to be quite analogous to the case involving the sentence "Venus is Venus". 
Salmon's response to this objection is, I think, unclear. It seems to me that there are at least two different arguments which he might be running. (In correspondence, Salmon has informed me that the second of these arguments is "more or less" his, while the first is definitely not. However, I still think that he does sometimes make use of something very much like the first argument.)

(i) First, he might be arguing as follows: Mere use of the description "the length of stick $\mathrm{S}$ at $\mathrm{t}_{\mathrm{o}}$ " in the fixing of the reference of the term "one metre" cannot provide one with knowledge about that length. True, it may allow you to form thoughts directly about that length -- i.e. the objection is not that the mere use of the description is insufficient to provide a way of taking the length; but it cannot provide you with the knowledge that one metre is this very length (here, the reader should imagine that I am pointing to -- an instance of -- the length one metre). "Notice that someone who has heard of the stick S but has not yet seen it could still introduce the term "one metre" by means of the description "the length of stick $\mathrm{S}$ at $\mathrm{t}_{\mathrm{o}}$ ". If the reference-fixer in this case has a wildly mistaken impression as to S's actual length ... or has no opinion whatsoever regarding S's length ... it would clearly be incorrect to describe him or her as knowing a priori of the length one metre that $S$, if it exists, is exactly that long at $\mathrm{t}_{\mathrm{0}}$."18 $^{18}$

This would be a strange argument for Salmon to use. One of his main theoretical claims is that one believes a singular proposition provided that there is a way of taking the proposition under which one believes it -- i.e. even if there are other ways of taking the proposition under which one fails to believe it. So how can "wildly mistaken impressions about S's actual length" be to the point? All that matters is that the reference-fixer should think (correctly) of the length one metre that it is the length of stick $S$ at $t_{0}$-- but surely the reference-fixer can do this even if s/he also has a wildly mistaken impression of the length of stick S! 
(ii) Second, Salmon might be arguing that, in fact, mere use of the description is insufficient to provide a way of taking the length. However, it seems to me that such an argument would simply trade on a confusion between linguistic descriptions and ways of taking objects. On the one hand, Salmon holds that ways of taking objects are not linguistic descriptions. So, of course, the mere possession of a linguistic description does not constitute possession of a way of taking an object. But, on the other hand, since Salmon holds that ways of taking are naturally associated with pieces of language, it remains to be demonstrated that there is no way of taking the length one metre which is naturally associated with the description "the length of stick $\mathrm{S}$ at $\mathrm{t}_{\mathrm{o}}$ ", and yet which furnishes the materials for an a priori apprehension of the contingent proposition that the length of stick $\mathrm{S}$ at $\mathrm{t}_{\mathrm{o}}$ is one metre. ${ }^{19}$

The chief problem here is that Salmon provides no positive characterisation of ways of taking. There is nothing in his work which would enable us to answer the question whether there can be a way of taking the length one metre which "builds in" the descriptive content that it is the length of stick $S$ at $t_{0}$, and yet which does not involve sensory experience of that length in such a way that that experience would necessarily be said to be playing a justificatory role in knowledge of the singular proposition that one metre is the length of stick $\mathrm{S}$ at $\mathrm{t}_{0} \cdot{ }^{20}$ Or rather, there is nothing in his work which enables us to answer the question why he takes it that there cannot be a way of taking the length one metre which "builds in" the descriptive content that it is the length of stick $\mathrm{S}$ at $\mathrm{t}_{0}$, and yet which does not involve sensory experience of that length in such a way that that experience would necessarily be said to be playing a justificatory role in knowledge of the singular proposition that one metre is the length of stick $\mathrm{S}$ at $\mathrm{t}_{\mathrm{o}}{ }^{21}$

The thought which Salmon seems to be resisting is that there are contingent propositions which one can know a priori provided that one has the right sort of way of taking the objects (and properties?) which are the constituents of those 
propositions. For the paradigmatic case of a proposition which can be known a priori -- the proposition which is the content of the sentence "Hesperus is Phosphorus" -any way of taking the planet Venus can form the basis for a way of taking that proposition under which it can be known a priori. But, if the proposition which is the content of the sentence "One metre is the length of stick $\mathrm{S}$ at to" could be known $a$ priori, it would only be given a very special way of taking the length one metre.

Evidence that Salmon's thought is tending in this direction is provided by his recent arguments against the contention that the proposition which is expressed by one's utterance of the sentence "I am here now" is a proposition which one can know a priori. $^{22}$

In defence of this claim, Salmon provides two arguments. Firstly, he says that "it would be decidedly mysterious if one could know of one's current location, without the slightest experiential contact with one's surroundings, that one is at that location. There is no like mystery in the fact that one can know without such contact that one is wherever one is, and that the sentence "I am here now" is therefore true with respect to one's context (whatever that may be)". ${ }^{23}$ Secondly, Salmon claims that it is possible for the proposition expressed by an utterance of "I am not here now" to be true -- e.g. when a recording of an utterance of "I am not here now" is re-played in the absence of the person whose made the recorded utterance: "I believe that this example is best thought of as a genuine case of assertion in absentia, in which the agent of the context is (just as he or she says) not present at the context of his or her speech act (and, indeed, is generally not even aware at the time of performing it).”24

It seems that Salmon's arguments against the claim, that the proposition which is expressed by one's utterance of the sentence "I am here now" is a contingent proposition which can be known a priori, naturally extend to arguments against the further claim, that the proposition which is expressed by one's utterance of the 
sentence "I exist" is a contingent proposition which one can know a priori. Firstly, there is nothing special about the role of spatial location in the first argument which Salmon gives. It would be equally as mysterious that one could know of the current time that it is the current time in the absence of any experiential link to that time, or that one could know of oneself that that person is oneself in the absence of any experiential link to that person. There might be no sense in which one knows who one is other than "whoever am I", and no sense in which one knows what time it is other than "whatever time it is". Of course, in having a thought and then reflecting upon the occurrence of that thought, one might also come to think of oneself as "the one having these thoughts", and of the present time as "the time at which these thoughts are occurring" -- but, equally, one could then come to think of one's present location as "the place at which the subject of these thoughts is located". Secondly, a recording of an utterance of "I do not exist” could be re-played at a time after the death of the person who made the utterance -- i.e. in a context in which the referent of the term "I" no longer exists. In that case, it seems that Salmon's earlier argument yields the conclusion that this would be a case of true assertion in absentia of the sentence "I do not exist". ${ }^{25}$

However, even though Salmon is now disposed to reject the claim that the semantic properties of the indexicals "I", "here", and "now" can furnish the materials for $a$ priori knowledge of contingent propositions, he continues to maintain that there are contingent propositions which can be known a priori. For example, he holds that the proposition which is the content of any instance of the schema "S iff actually S", for any sentence "S" which has a contingent proposition for its content, will be both contingent and knowable a priori. But the question now arises whether this contention is consistent with Salmon's arguments concerning the status of the proposition which is the content of an utterance of "I am here now". 
According to Salmon, the semantic content of a tokening of the word "actually" in a context $c$ is the property of being true in $w$, where $w$ is the possible world in which w occurs. But, to parallel the first of Salmon's earlier arguments, it would be decidedly mysterious if one could know of the actual world a priori that it is the actual world, in the absence of experiential contact with the actual world. Just as it might be that the only sense in which one's use of the indexical "here" yields a way of taking one's current location is as "whichever location I am currently in", so it might be that the only sense in which one's use of the indexical "actually" yields a way of taking the actual world is as "whichever world I am in". ${ }^{26}$ In each case, further experiential contact would be required to justify the claim that there is a more substantial sense in which one has knowledge of the object in question. ${ }^{27}$

I conclude (i) that if Salmon' arguments about the status of propositions expressed by utterances of the sentence "I am here now" are correct, then those arguments can be extended to support the view that there are no contingent propositions which can be known a priori; and (ii) that if those arguments are correct, then they support the general thesis that there are no contingent propositions which one can know a priori only provided that one has the right sort of way of taking the objects (and properties?) which are the constituents of those propositions.

But are those arguments correct? Well, surely the second argument should be resisted. Suppose we grant the controversial claim that the case which Salmon describes is best thought of as a case of assertion in absentia. Then it will follow that there are contexts in which I utter the words "I am here now", and yet in which the content of that utterance is not a true proposition. But this putative fact is simply irrelevant to the question whether there are contingent propositions which can be known a priori. On the one hand, in order to show that one can never know a priori the proposition which is the content of one's utterance of the sentence "I am here now", one would need to show that there is room for doubt in every context of 
utterance. But there is nothing in Salmon's argument which does this. And, more importantly, on the other hand, the crucial question is not about the content of utterances, but rather about ways of taking the content of those utterances. Even if it is true that one's utterances can "issue" from contexts in which one is absent, it is surely not true that one's thoughts can do this. Given that I have "indexical" ways of taking myself, my present location, and the present time, then I have the materials for an a priori grasp of the proposition that I am here now, whether or not some of my utterances of the sentence "I am here now" can fail to be true.

So, the success of Salmon's arguments turns on the question whether there can be "indexical" ways of taking objects which do not involve experience in such a way as to disqualify the grasp of proposition under those ways of taking from being a priori. But, as I mentioned earlier, it is hard to know how to answer this question until one has a much clearer account of the nature of ways of taking objects. I must confess that I do feel some sympathy for Salmon's opinion -- i.e. I sometimes find myself inclined to assent to the claim that there can be no such ways of taking objects -- but I am far more certain that there is a clear need for analysis of, and argument about, conceptions of ways of taking objects. ${ }^{28}$

One might be tempted to defend the claim that there can be no "indexical" ways of taking objects in the following way: The supposition that there are such "indexical" ways of taking objects trivialises the notion of the contingent a priori -- for it turns out that every true contingent proposition can be known a priori. We are asked to suppose that, while the sentences "I am here now" and "Graham is in such-and-such a place at such-and-such a time" express the very same proposition, only the first sentence is naturally associated with "indexical" ways of taking the constituents of that proposition in such a way that those ways of taking can be combined to form a way of taking the proposition under which it can be known a priori. But now consider such sentences as: "Peter is 5'9" tall", "Mary was born in Seattle", and 
"Water runs downhill". Corresponding to these sentences, there are sentences like the following: "Peter is dthat(the height which Peter is) high"; "Dthat(the one who is identical with Peter and who is 5'9" tall) one is 5'9" tall"; "Mary was born in dthat(the place in which Mary was born) place"; Dthat(the one who is identical with Mary and who was born in Seattle) one was born in Seattle"; "Dthat(the stuff which is identical with water and which runs downhill) stuff runs downhill"; and "Water runs in dthat(the direction in which water runs) direction". ${ }^{29}$ According to Salmon's semantic theory, the true sentence "Peter is 5'9" tall" and the sentence "Dthat(the one who is identical with Peter and who is 5'9" tall) one is 5'9" tall" have the very same content. But, if there can be an "indexical" way of taking my present location which is naturally associated with the word "here", and which does not involve experiential contact with that location in such a way as to rule out the possibility that it might play a crucial role in the acquisition of a priori knowledge about that location, then surely there will be an "indexical" way of taking Peter which is naturally associated with the expression "Dthat(the one who is identical with Peter and who is 5'9" tall) one", and which does not involve experiential contact with Peter in such a way as to rule out the possibility that it might play a crucial role in the acquisition of a priori knowledge about him. ${ }^{30}$ At the very least, it is prima facie plausible that the concession that there are "indexical" ways of taking objects will lead to a trivialisation of the notion of the contingent a priori. ${ }^{31}$

Perhaps this argument would provide Salmon with reason to reject the claim that there are "indexical" ways of taking objects. However, it is not clear that all theorists who accept the existence of singular propositions ought to be persuaded by it. For -to turn to an objection which many would wish to put to Salmon -- surely it is the case that a priority is primarily a property of propositions under ways of taking those propositions. That is, surely the trivialisation argument can be avoided, even if one accepts the existence of "indexical" ways of taking objects, provided that one gives up the idea that a priority is primarily a property of propositions. 
To this objection, Salmon might object that one gets a simpler total theory (of semantics and philosophy of mind) if one adopts his theory. However, as I have argued elsewhere, there is a total theory (of semantics, pragmatics, and philosophy of mind) which is certainly no more complex than his, and yet which also does not have the prima facie counterintuitive consequences of his theory.

On this alternative view, ways of taking objects (and properties?) do have a role to play in semantics. In particular, on the version of the theory which I prefer, the semantic contents of sentences embedded in the scope of verbs of propositional attitude include both singular propositions and contextually supplied conditions or restrictions on ways of taking objects and properties. ${ }^{32}$ However, for present purposes, it is probably enough to observe that almost any theory which rejects substitutivity principles for co-referring names, indexicals and demonstratives will be able to avoid the trivialisation which threatens to undermine Salmon's account of the contingent a priori . Most theories which eschew those substitutivity principles, and yet which also provide an important role for singular propositions, will have the resources to allow one to say that, while it is (strictly speaking) true that I can know $a$ priori that dthat (the one who is identical with Peter and who is 5' 9" tall) one is 5' 9” tall, it is (strictly speaking) false that I can know a priori that Peter is 5' 9” tall. ${ }^{33}$

So, in sum, it seems to me that there are two lines which Salmon could take. Either he could deny that there are "indexical" ways of taking propositions, and hence deny that there are any contingent propositions which can be known a priori; or he could accept that there are "indexical" ways of taking propositions, but modify his account of the nature of a priority and the semantics of propositional attitude ascriptions in order to avoid the trivialisation argument given above. ${ }^{34}$ Given the slack in our intuitions about de re thoughts, and given the difficulty of providing any interesting analysis of ways of taking propositions, I am inclined to think that there is some 
reason to prefer the second option -- i.e. pending further argument, there is reason to accept that there can be a priori knowledge of contingent propositions. But, at best, this conclusion is quite tentative. ${ }^{35}$

Moreover, even if one were to accept this conclusion, one might wonder about the wider philosophical import of the putative fact that there are numerous (strictly speaking) true sentences of the form It can be known a priori that $p$ for which the embedded (singular) proposition is contingent. I suspect that there would be almost none. For it seems clear that all such sentences would involve some sort of linguistic indexicality (explicit indexicals and demonstratives, dthat-type operators, etc.) which is intimately related to the epistemic link between cognitive subjects and the propositions in question. But then, once this fact is acknowledged, what further metaphysical capital would there be to be gained? In particular, there would be no very strong sense in which one possessed a priori knowledge about contingent matters of fact.

A similar point can be made about the putative fact that there are numerous (strictly speaking) true sentences of the form It can only be known a posteriori that $p$ for which the embedded (singular) proposition is necessary. (That there are such sentences does not follow immediately from the acceptance of a theory which rejects substitutivity principles for co-referring names, indexicals and demonstratives in the scope of verbs of propositional attitude. However, if the principle of the necessity of identity is also accepted -- a point on which Salmon and I agreee -- then it does follow that Kripke was also right about the necessary a posteriori.) In these cases, it is the fact that there can be different modes of presentation of a single object -- in circumstances in which further information is required before it can be determined that the different modes of presentation are, in fact, modes of presentation of a single object -- which is made salient. Again, I doubt that anything of importance now follows. 
Of course, these results might be of historical interest. Perhaps Descartes' Cogito is best understood in terms of the above characterisation of the contingent a priori. But, if so, that supports my further claim -- for, in that case, nothing of any importance would follow from Descartes’ Cogito.

IV

Since the theory of propositional attitude ascriptions which I favour is controversial, I would like to indicate (in closing) how much of what I have argued for in this paper is independent of that theory. I claim that Salmon's views either lead to the view that there are no contingent propositions which can be known a priori, or else lead to a trivialisation of the notion of knowability a priori. However, on a fairly minor restructuring of his theoretical apparatus ${ }^{36}$, both of these results can be avoided -- but at the cost of agreement with Kripke both about the necessary a posteriori and the contingent $a$ priori. In my view, such a minor restructuring of Salmon's views leads to a correct account of propositional attitude ascriptions -- and hence to a defence of Kripke's original claims. However, those who favour a more radical rejection of Salmon's account of propositional attitudes ought not to be persuaded, that Kripke is right, by anything which I have argued here.

\section{Postscript A}

Since I wrote this paper, I have receieved some comments from Nathan Salmon. In particular, I have learned that he would respond to my main complaint -- viz. that his failure to say anything positive about the nature of ways of taking makes is hard to see why he refuses to countenance descriptional ways of taking -- as follows: 
The method which he follows develops from (what he claims is) a straightforward application of (i) the distinction between the general belief that there are spies and the particular belief that so-and-so is a spy; and (ii - a particular case of (i)) the distinction between the de dicto belief that the shortest spy is a spy and the de re belief of the shortest spy that s/he is a spy. "Consider someone who has never experienced how long $\mathrm{S}$ is, someone who knows of the stick but has no idea how long it might be (shorter than one inch? longer than one mile?) Ask yourself whether such a subject knows of the length -- de re -- that $\mathrm{S}$ is exactly that long. Obviously not. The subject is not even thinking of the length in question, de re, that $\mathrm{S}$ is exactly that long. ... My methodology here builds on these sorts of intuitions, taking them to be Gospel."

It seems to me that this response simply begs the question. A defender of descriptional ways of taking will want to insist that the sentences "The reference-fixer knows a priori that the length of stick $\mathrm{S}$ at $\mathrm{t}_{\mathrm{o}}$ is one metre" and "The reference-fixer knows a priori that the length of stick $\mathrm{S}$ at $\mathrm{t}_{\mathrm{o}}$ is exactly that long" differ in truthvalue precisely because the expression "one metre" (in the context of the example) is naturally associated with a descriptional way of taking whereas the expression "exactly that long" is not naturally associated with a descriptional way of taking. So, even granted the intuitions on which Salmon builds his theory, I don't see that we have advanced any further than Salmon's brute intuition that there are no (cannot be any) descriptional ways of taking. ${ }^{37}$ The defender of descriptional ways of taking concedes virtually everything that Salmon wants to say -- e.g. that the reference-fixer does not know a priori that the length of stick $\mathrm{S}$ at $\mathrm{t}_{\mathrm{o}}$ is exactly that long -- but insists, nonetheless, that the reference-fixer does know a priori that the length of stick $\mathrm{S}$ at $\mathrm{t}_{\mathrm{o}}$ is one metre. 
There is also a methodological point which arises. Salmon takes as basic certain intuitions -- e.g. that the reference-fixer is not acquainted with, does not have knowledge of, does not have a de re thought whose content is, the singular proposition which is the content of the relevant sentence -- and uses these intuitions to decide the truth-values of certain propositional attitude ascriptions. But all of these intuitions involve controversial notions -- "de re", "about", "of", "knows which" -which are very dubiously suited to the task of serious theorising. I do not dispute that these terms have legitimate and natural uses -- though I do have doubts whether there is a natural (pre-theoretical) practice of making de re attitude ascriptions -- but I suspect that these uses are highly context-sensitive and interest-relative. And, in particular, I would suggest that theories which invoke singular propositions would do better to use untutored propositional attitude ascriptions (in given contexts) as a guide to the conditions under which subjects are acquainted with (have de re thoughts about, etc) those singular propositions. ${ }^{38}$

\section{Postscript B}

Since I wrote the previous postscript, I have obtained a copy of a very recent article by Salmon ${ }^{39}$. It seems to me that, inadvertently, Salmon now concedes that the meterstick and Newman 1 cases provide instances of the contingent a priori.

In the recent paper, Salmon draws a distinction between pure semantics and applied semantics. He suggests that the following are facts of pure semantics: that the definite description "the inventor of bifocals" refers to the inventor of bifocals; that the name "Tully" refers to Tully. And he suggests that the following is a fact of applied semantics: that the definite description "the inventor of bifocals" refers to Benjamin Franklin. He goes on to apply this distinction to the case of Newman 1: 
Given the manner in which the reference of "Newman 1" is fixed, the fact that "Newman 1" refers to the first child born in the 22nd. Century, and hence also the resulting fact that the "Newman 1" sentence is true, do indeed seem to be facts of pure rather than applied semantics. ... Interestingly, the further fact that "Newman 1 ” refers to Newman 1 seems to be a fact of applied semantics rather than pure semantics, since it obtains only by virtue of the non-linguistic fact that Newman 1 (i.e. that very future person) will be the first child born in the 22nd. Century. ${ }^{40}$

But, insofar as I understand the distinction between pure and applied semantics, it seems to me that it must be a fact of pure semantics that "Newman 1 " refers to Newman 1. After all, the fact that "Tully" refers to Tully obtains only by virtue of certain non-linguistic facts -- e.g. that Tully (i.e. that very person) actually existed -and yet Salmon confidently declares that this is a fact of pure semantics. If it can turn out that some instances of the schema " $X$ " refers to $X$ do not express facts of pure semantics, then what reason do we have to say that the fact that "Tully" refers to Tully is a fact of pure semantics, and hence is knowable a priori $?^{41}{ }^{42}$

\footnotetext{
1Second Edition, Oxford: Basil Blackwell, 1980

${ }^{2}$ MIT Press, 1986. (The book is now published by Ridgeview Publishing Company, Atascadero CA.)

${ }^{3}$ Henceforth, I shall drop the explicit qualification "by creatures like us"; however, it is always implicitly assumed.
}

${ }^{4}$ Frege's Puzzle, pp.131-135. This view has odd consequences -- e.g. it forces Salmon to deny that all instances of the following schema have true propositions as their contents:

It is logically true that p iff "p" (as uttered in the present context) is logically true.

In Salmon's view -- as I understand it -- "logically true" is used in two different senses here, the first use being a merely derivative application to propositions.

Salmon says that a proposition is logically true with respect to a context if it is the content, with respect to that context, of a logically valid sentence in some possible language. And he then says that a proposition is logically true (simpliciter) if, for every possible context, it is the content, with respect to that context, of a logically valid sentence in some possible language. The point of this distinction is that, while certain indexical sentences such as "I exist" and "I am here now" are logically true (in Kaplan's logic of demonstratives), the propositions which they express on given occasions of utterance -- e.g. that Graham exists -- are merely contingently true, and hence not deserving of the appellation "logically true (simpliciter)".

However, one is tempted to object that every true singular proposition will be logically true with respect to a context. For suppose that we have a true singular proposition whose constituents are an 
object a and a (perhaps complex) property F. Suppose further that "the thing which is G" is a description which uniquely identifies a. Then (i) the expression "Dthat (the thing which is $G$ and F)" is a singular term which has as its content the object a, and the sentence "Dthat(the thing which is $G$ and

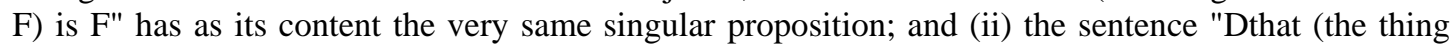
which is $\mathrm{G}$ and $\mathrm{F}$ ) is $\mathrm{F}^{\prime \prime}$ is logically true.

Now, this argument is a bit swift: for what if there were no thing which is both G and F? Surely the logically true sentence is rather this: "Dthat (the thing which is G and F), if it exists, is F". Well, surely that would be trouble enough! For we can still argue that any true singular proposition which contains the appropriate existential qualification is logically true with respect to a context -- a result which seriously trivialises the notion of logical truth with respect to a context. (The proposition expressed by the sentence "Graham, if he exists, wears glasses" is logically true with respect to a context.)

5I think that Salmon holds that the attributes which apply "primarily, and in the first instance" to sentences and sets of sentences are purely formal properties of sentences and sets of sentences. But it is clear that analyticity is not this sort of property of sentences and sets of sentences. (Of course, it is also clear that truth is not this sort of property of sentences and sets of sentences!) On the other hand, it seems wrong to think that analyticity is primarily a property of propositions -- and, moreover, Salmon's own explanation of analyticity is an explanation of a property which is possessed by sentences in virtue of the semantic properties of (some of) their constituent words. (Of course, there is some plausibility in the view that truth is a property which is possessed by sentences in virtue of the semantic properties of (some of) their constituent words!)

The view that logical attributes are purely fomal properties of sentences and sets of sentences is not without difficulties. The idea is that logical attributes are determined by holding the meanings of all purely logical expressions fixed, while allowing the meanings of all non-purely-logical expressions to vary across all admissible (semantic) interpretations. Of course, in any given admissible interpretation, the assignment to each expression will be constant -- i.e. semantic equivocation will not be allowed. Consequently, the notion of semantic interpretation still does some work here: sentences in which terms are given equivocal interpretations do not count as logical truths in virtue of their formal similarity to genuine logical truths. This suggests that, maybe, the logical properties should be taken to be mixed as well -- though in a different sense to that in which analyticity is a mixed property. Perhaps that would not upset Salmon too much; what his argument requires is only that a priority, necessity, etc. are primarily properties of propositions. (But cf. subsequent discussion in the text.)

One difficulty for this account of the logical attributes is that it relies upon a distinction between purely logical and not-purely-logical vocabulary. But it may not be very easy to draw this distinction -especially given the resources to hand. Moreover, it is very doubtful that, for example, classical firstorder logic can be derived on the basis of such a distinction. (There may be other difficulties as well. See John Etchemendy's Logical Consequence, Oxford: Oxford University Press, 1990 for further discussion.)

Perhaps Salmon might reply that he does not intend his claim about the primacy of logical attributes to be understood in the way I have suggested. (Note, in particular, that he nowhere commits himself to these ideas.) However, the difficulty then is that it is very hard to see what account could possibly be given of the nature of logical attributes. At the very least, he owes some further explanation -- and, if what I have said here is correct, that explanation will surely require some very fancy footwork. (In passing, I will also note that the elaborate semantic theory which Salmon has developed floats free of any accompanying proof theory. If -- for example -- we had a proof theory which was demonstrably sound and complete with respect to Salmon's semantics, then we would presumably have the materials for an account of the logical attributes of sentences.)

'In Frege's Puzzle, p.109, Salmon writes:"Must the mode of acquaintance be causal? Is any causal relation enough? (Consider the case of numbers and mathematical knowledge.) Is it enough simply to have heard the individual mentioned by name? Is it enough to be able to refer to the object? (Consider the shortest spy.) Is it enough simply to point at the object, without even looking to see what one is pointing at? Must one have some conception of what kind of thing the object is (a person, an abstract entity, etc)? Can one have mistaken opinions about the object? How many? Does one have to know who the individual is, or which object the object is, in some more or less ordinary sense of "know who" or "know which"? Must one know some feature or characteristic of the object or individual that 
distinguishes it (or him or her) from all others? Is it sufficient simply to know some distinguishing feature or characteristic (i.e., is what Russell called "knowledge by description" always enough)?"

${ }^{7}$ See the discussion at pp.127-128. ("[I]t might also turn out that there are .. ways of taking a proposition .. such that there is no proposition which they are ways of taking.")

8See "Existence" in J. Tomberlin (ed.) Philosophical Perspectives I, Ridgeview: Atascadero CA, 1987, pp.49-108, especially Section VI, pp.90-98. It may be that this is not really a different view -- for it may be that the view to which Salmon referred, albeit briefly, in Frege's Puzzle, was the view that there is no existent proposition which is the content of the sentence "Santa Claus has a white beard".

${ }^{9}$ Salmon's views on this topic are further elaborated in "Existence", op. cit. It seems to me that, in order to take his preferred option seriously, one needs to accept his controversial claims about quantification and existential commitment -- e.g. that the quantifiers of English are typically actualist and presentist. After all, it is tempting to respond that if Santa Claus is a fictional character, then surely it follows that Santa Claus exists! Although I cannot hope to argue this here, I think that this is the correct response to make to Salmon's preferred suggestion -- and, hence, that he must choose from between (i) and (ii). Moreover, it may be that I shall need to make use of this observation -- for I shall rely on the assumption, that if stick S does not exist then "stick S" is a genuinely non-referring term, at subsequent points in the paper (especially in footnote 14).

${ }^{10}$ In other words, I am making the following two suggestions: (i) that we should take Salmon's use of the expression "strictly speaking" to be intended to effect the cancellation of the conventional (or generalised) implicatures which he claims attach to propositional attitude ascriptions; and (ii) we should also take it that Salmon's intention is that the usual implicatures of propositional attitude ascriptions are lost in context in which we are speaking strictly.

${ }^{11}$ Perhaps this is too swift. After all, Naming And Necessity is intended to be a popular expression of Kripke's ideas. It is not clear that, by Salmon's lights, Kripke really does make a mistake when, in the course of his lecture series, he says that (maybe) one can only know a posteriori that Hesperus is Phosphorus. Speaking loosely -- i.e. not strictly -- one can only know a posteriori that Hesperus is Phosphorus, even though it is necessarily the case that Hesperus is Phosphorus! So, speaking loosely, we certainly do have here an example of the necessary a posteriori. But, of course -- if Salmon is correct -- it is also true that, speaking strictly, we do not have an example of the necessary a posteriori.

${ }^{12}$ There is an important methodological assumption -- a version of methodological conservatism -which I would invoke against Salmon at this point. Ultimately, the data for a philosophical theory of a language are the assertions made by ordinary speakers of the language. Sometimes, those assertions fail -- e.g. because they are false, or conversationally inappropriate. However, one ought to believe that the norms for the language are embedded in the total practice of those speakers: i.e. (other things being equal) one ought not to believe that, for some kind of commonly used linguistic construction, assertions which involve them almost always fail and yet expert participants in the practice take it that the assertions in question very often succeed. Thus -- unless a compelling case can be made for the claim that other things are not equal -- one ought not to believe a theory which says that assertions involving term identities embedded in propositional attitude ascriptions almost always fail. It is much more reasonable to believe that a philosophical theory of meaning which has this consequence is false than it is to believe that the practice in question is radically defective.

${ }^{13}$ In this section, I shall follow the expanded discussion of Salmon's "How To Measure The Standard Metre”, Proceedings Of The Aristotelian Society, 1988, pp.193-217. The argument is essentially the same as that given in Frege's Puzzle, but with more of the details filled in.

${ }^{14}$ Strictly, the sentence which Salmon discusses contains an existential qualification, viz: The length of stick $S$ at to, if it exists, is one metre. The intuitive reason for the inclusion of the qualification is that one could not know a priori that S exists. However, it is not immediately clear how the addition of the existential qualification can help with the problem.

On the one hand, if we adopt the view that sentences which contain non-denoting terms do not express propositions, then the addition of the qualification cannot help to ensure that the new sentence does 
express a proposition. On this view, if $\mathrm{S}$ does not exist, then the sentence The length of stick $S$ at to, if it exists, is one metre does not express a proposition -- and hence does not express a truth. So, if the reference-fixer cannot know a priori that S exists, then the reference-fixer cannot know a priori that the sentence The length of stick $S$ at to, if it exists, is one metre expresses a (true) proposition, and (hence?) cannot know the proposition expressed by that sentence a priori even in the case in which it does express a proposition.

On the other hand, if we adopt the view that sentence with non-denoting terms express merely possible propositions, then it is tempting to suppose that the addition of the qualification is redundant -- for, whether or not stick S exists, the length of stick S at to will be one metre! In arguing thus, one would need to rely on the assumption that even a merely possible stick has a length. However, Salmon would dispute this assumption: in his view, a merely possible stick only has the dispositional property that it would have (such-and-such?) a length if it existed.

So, given all of Salmon's theoretical commitments, we do have an explanation of the need for -- and the utility of -- the existential qualification. On his view, the sentence The length of stick $S$ at to, if it exists, is one metre is guaranteed to express a proposition. However, what sort of proposition it expresses depends upon the existence or non-existence of stick S.

Now, it is surely a controversial question whether we wish to adopt Salmon's account of the properties which are possessed by merely possible objects. In particular, it is not clear what account is to be given of the distinction between the properties which can, and the properties which cannot, be possessed by merely possible objects. (Meinongians face a similar difficulty in the case of the distinction between nuclear and non-nuclear properties.) Moreover, there is an alternative which a proponent of singular propositions can adopt, viz: that, in fact, even though one cannot know a priori whether stick S exists, nonetheless, one can know a priori the proposition which is expressed by the sentence The length of stick $S$ at to is one metre, provided (i) that stick $S$ does exist, and (ii) that one does have a way of thinking of the stick. In order to make this suggestion work, one will need to deny something like the KK-principle for a priori knowledge -- for, to speak loosely, it may well be that one is unable to tell whether or not one possesses a way of thinking of the stick, even when one does in fact possess such a way of thinking of the stick. It is not obvious to me that this alternative is less attractive than the one which Salmon adopts.

In sum, then: I think that it remains to be argued that the existential qualification is required. I shall omit it; but those who feel that it ought to be included should pretend that it has been included. None of the later arguments of this paper will turn on the choice which is made here.

(N.B. The discussion of this footnote ignores the possibility that there are "gappy" propositions. On such a view, and given a suitable three-valued logic, it would turn out that the simple sentence does not express a true proposition in circumstances in which $S$ fails to exist, but that the qualified sentence always expresses a true proposition in the context of the reference-fixer. I do not know whether this suggestion should be preferred to the third alternative discussed above.)

${ }^{15}$ How To Measure The Standard Metre", p.198.

Notice that, in the quoted paragraph, Salmon stresses that one needs experience in order to know that S is precisely, or exactly, one metre in length. This may seem odd. Indeed, one might be tempted to object that, for any object apart from S, the most that any purely a posteriori experience could reveal is that it is roughly, or approximately, one metre in length. However, Salmon's point is not that reference-fixer comes to know the length of the metre stick by purely a posteriori means; rather, the point is that that knowledge is arrived at by a joint process: the reference-fixer must both make the stipulation and (simultaneously?) see -- or otherwise have de re contact with -- the length of the metre stick. In general -- as Salmon himself notes -- our standards for knowing the lengths of things are sensitive to interest and contextual factors; but, for any ordinary standards of precision, the referencefixer can satisfy them trivially in the case of the metre stick.

Perhaps there is still a problem here. Suppose that I introduce the term "alphon" with the stipulation that one alphon is the distance from here to Alpha Centauri now; and that I also introduce the term "sirion" with the stipulation that one sirion is the distance from here to Sirius now. Even if I make these stipulations while looking at the appropriate objects, it seems that it would be wrong to say that I 
see the lengths involved. (In particular, no one would say that, just by looking, it is possible to determine which length is greater. But do I really see either length if I cannot judge which is longer? Would I see the length of the metre stick if the stick were located in one of those rooms which distorts perspective, and which created the impression that it was the length of a cricket pitch?) Yet, on the other hand, it seems to me that, even in my present state, I do know that one alphon is one alphon, and that one sirion is one sirion. And, moreover, it also seems to me that I know that one alphon is the distance from here to Alpha Centauri now, and that one sirion is the distance from here to Sirius now. (Even if we add to this example the further information that I know -- independently -- that the distance from here to Alpha Centauri is roughly four light years, my epistemic status would surely be unchanged: for one alphon is only roughly four light years. The same would be true in the case of the metre stick if we added the further information that I know -- independently, as a result of being told -that the length of the metre stick is between three and four feet.)

I suppose that Salmon could just bite the bullet here, and insist that there is no sense in which -- no context, no relevant set of interests, with respect to which -- I know how long one alphon is, unless there is some independent information which I have which derives from that length. However, I find it hard to see how there could be a non-question-begging argument in defence of that view.

${ }^{16}$ Perhaps I ought rather to say that it is constitutive of the way in which the reference-fixer takes the length one metre that $\mathrm{s} /$ he takes it to be the length of stick $\mathrm{S}$ at $\mathrm{t}_{\mathrm{o}}$. Part of the difficulty here is that we know so little about the nature of ways of taking objects and propositions. (Cf. further comments in the text.)

17"How Not To Become A Millian Heir", Philosophical Studies, Vol 62, 1991, pp.165-177, at p.171.

18"How To Measure The Standard Metre", pp.198-199.

${ }^{19}$ Here is another reason for thinking that the objection better not be that the mere use of the description is insufficient to provide a way of taking the length: Consider the expression "one light year". There is considerable (!) plausibility in the view that the reference of this expression is fixed by the description "the distance which light travels in one year". Now, consider the proposition which is expressed by the sentence "One light year is one light year". It seems to me that it would be wrong to suggest that I do not know this proposition -- and, indeed, that it would be wrong to suggest that I do not know this proposition a priori. But, of course, any independent impression which I have of this length is very likely to be wildly mistaken -- it is, after all, a very great length (one of which it is impossible to form any directly experiential impression). Perhaps it might be objected that I can only know this proposition if I have some independent way of taking the length -- e.g. as $\mathrm{N}$ metres, for some very large $\mathrm{N}$. But I doubt that my grasp of "N metres", insofar as it is a conception of a particular length, will be any more accurate than my grasp of "one light year" (i.e. it seems to advance me no further in my understanding of how far it is). Of course, this argument relies on the controversial thought that a way of taking a length must furnish an accurate conception of how long it is. Since Salmon himself seems to make similar assumptions, there may be sufficient material for an objection here -- but what is really needed is more discussion of the connection between knowing which (who, how, etc) and de re thought.

Despite the above considerations, there is some evidence that Salmon does think that the mere use of the description is insufficient to provide a way of taking the length -- even if it is supplemented with experience of the stick $\mathrm{S}$ which provides no further information about its length. (Suppose that the reference-fixer is blind, but that s/he introduces the description while holding on to the stick S.) In particular, he claims that the reference-fixer does not know of the length one metre that the use which s/he makes of the expression "one metre" refers to it -- and, indeed, that there is a strong sense in which the reference-fixer does not understand the use which s/he makes of the expression "one metre".

${ }^{20}$ One consequence of Salmon's neglect of this question is that it makes it unclear how to assess his criticisms of the work of theorists who suppose that there can be demonstrative modes of presentation. In particular, Salmon's criticisms of Gareth Evans' remarks about the name "Julius" -- presented, e.g., in Evans (1979) "Reference And Contingency", Monist, pp.161-189, and in Evans (1982) The Varieties Of Reference, Oxford: Oxford University Press -- rely on a refusal to take seriously the idea that one's use of a "descriptive name" might be associated with a descriptional mode of presentation of 
the referent of that name. See, in particular, p.198n7 of "How To Measure The Standard Metre"; also, see the next footnote in the present paper. (In Salmon's own terminology, the "description" associated with a "descriptional" name is not part of -- and nor does it determine part of -- the semantic value of the name; rather, it is part of the way of taking (the referent of the name) which gets associated with the name in the context in question.)

${ }^{21}$ Salmon's discussion of "descriptive names" provides a clear illustration of the fact that he does think that it is impossible for there to be a way of taking the length one metre which "builds in" the descriptive content that it is the length of stick $\mathrm{S}$ at $\mathrm{t}_{\mathrm{o}}$ and yet which does not involve sensory experience of that length in such a way that that experience would necessarily be said to be playing a justificatory role in knowledge of the singular proposition that one metre is the length of stick $\mathrm{S}$ at $\mathrm{t}_{\mathrm{O}}$. Consider, for example, the well-known case in which the name "Julius" is introduced using the description "the actual inventor of the zip" to fix its referent. Salmon claims (i) that the proposition which is the content of the sentence "The inventor of the zip, if there is one, is the actual inventor of the zip" is indeed contingent and a priori, but that it does not involve de re knowledge about Julius; and (ii) that the proposition which is the content of the sentence "Julius invented the zip, if anyone did" is contingent, but can only be known a posteriori.

22These arguments may be found in "How Not To Become A Millian Heir", pp.171-172.

23"How Not To Become A Millian Heir", p.171.

24"How Not To Become A Millian Heir", p.171

${ }^{25}$ Salmon notes this consequence of his argument in "How Not To Become A Millian Heir", p.176n21.

${ }^{26}$ Notice that it is crucial to distinguish between two different properties here. First, there is the property of being true in a, where a is the actual world; and, second, there is the property of being true in $w$, where $\mathrm{w}$ is whichever world the utterance under evaluation occurs in. I think that it is plausible to make the following two claims: (i) in order to know that a proposition involving the first of these properties is true, one must know which world is the actual world (according to the relevant contextrelative and interest-relative standards); and (ii) it is appropriate to say that one knows that a proposition involving the second property is true if all that one knows is that a sentence has a true proposition as its content and, as a matter of fact, that property is involved in the proposition in question (even if there is no more robust sense in which one knows which world is the world in which one is located). Thus, it seems to me that, if the arguments discussed in the text were correct, then Salmon ought to say (i) that all that one knows a priori are necessary propositions about which sentences express true propositions; and (ii) that the related contingent propositions can only be known a posteriori (given suitable de re connections with the actual world).

${ }^{27}$ Of course, on any standard conception of possible worlds, there is no plausible parallel to the second of the arguments which Salmon gives -- for it is part of those standard conceptions that there can be no travel between worlds. However, this only shows that there could be no false assertion in absentia of sentences of the form "S iff actually S". (If, per impossible, largish chunks of possible worlds could detach from some worlds and reattach to others, then it might be possible to tell a story according to which there is a false assertion in absentia of a sentence of the form "S iff actually S".)

${ }^{28}$ There are other reasons why Salmon should pay more attention to ways of taking objects. Consider, e.g., the following passage from Frege's Puzzle (n.5, pp.175-176):

"Consider then the English sentence "Aristotle was Aristotle". Is it a logical truth (a valid theorem)? Well, strictly speaking it is ambiguous. Let us disambiguate it. Suppose that the first occurrence of "Aristotle" refers to the philosopher and the second to the shipping magnate. Then it is surely not a logical truth, since it is false. Of course, if instead both occurrences of "Aristotle" had referred to to the philosopher, then the expression form would have been a logical truth. Now, suppose once again that, though we do not know it, the philosopher of antiquity did not die in ancient times as we think, but went into hiding, discovered the philosopher's stone which slows down the ageing process, and emerged in the twentieth century as the powerful shipping magnate. What then of the logical status of the disambiguated sentence in question? Can it be a logical truth, with the first occurrence of 


\begin{abstract}
"Aristotle" intended to apply to the philosopher and the second occurrence to the shipping magnate, even though no one now alive who understands the sentence as it is intended is in a position even in principle to recognise it as a logical truth. Can a sentence be a simple logical truth of the form $a=a$ even though an ideally perfect logician who fully understands the sentence would have to reckon it not logically valid? .. Or is it really logically valid after all? If so, what differentiates it from the disambiguated sentence with both occurrences of "Aristotle" intended to apply to the same person? Surely the latter sentence is a logiocal truth. How can the former, if true, differ at all in logical status from the latter?"
\end{abstract}

Response: A sentence will be taken to be a logical truth only provided that the terms which it contains are taken in the same way throughout. Contra the view which Salmon espouses, it is a mistake to say that logical properties are primarily properties of sentences if this claim is taken to mean that logical properties are merely formal properties of sentences. What is crucial is that sentences only have logical properties under interpretations -- and that means, not merely that there must be an assignment of objects to the singular terms in the sentences, but also that there must be an assignment of ways of taking those objects to the subjects who are uttering the sentences. (Notice that I do not say that there must be an assignment of ways of taking objects to the singular terms involved; I take it that that thought has been thoroughly refuted by Kripke's arguments in Naming and Necessity.)

One consequence of this response to Salmon's argument is that it seems most plausible to say that logical properties are primarily properties of sentences under ways of taking the propositions which are the contents of those sentences. That is -- cf. footnote 5 above -- it is not correct to say that logical properties are mixed, if that is merely taken to mean that logical properties are primarily properties of sentences under semantic interpretation (i.e. under interpretations in the standard logical sense). Moreover, it also suggests that it might be fruitful to investigate the question whether, in the primary sense, logical properties are properties of ways of taking propositions -- though, of course, as I have already noted in connection with some other important questions, an answer to this question will require a full and careful account of the nature of ways of taking propositions.

${ }^{29}$ The last example may seem suspect, since one cannot really demonstrate the direction in which water runs. However, in order to determine whether this example should be rejected, we first need to determine whether there can be different ways of taking properties -- and, if so, whether there can be "indexical" ways of taking properties. Very little attention has been paid to questions about the nature of properties in the literature on singular propositions -- so it is hard to know how one should even begin to think about these issues.

${ }^{30}$ In this paragraph, I have ignored complexities involving the possible non-existence of the subject of the various sentences. Cf. the discussion of footnote 4 above for the obvious amendment. Even with the amendment, the argument still produces a serious trivialisation of the notion of the contingent $a$ priori. Moreover, it is not clear that the need for this amendment produces a serious disanalogy between sentences involving "dthat" and sentences involving more familiar indexicals ("I", "here", "now").

In the earlier discussion, I have taken for granted the assumption that any tokening of the word "here" will have a definite content (the place of the context of utterance). But there are several reasons for being sceptical about this assumption. (i) It could be argued that a tokening of the word "here" only has a content relative to a choice of a spatio-temporal frame of reference (i.e. relative to a choice which cannot be made a priori). When I say that I am "here" now, I could mean at such-and-such a place on the surface of the earth, or I could mean at such-and-such a place in the Milky Way, or I could mean at such-and-such a location from the centre of the universe, etc. That these are different locations is shown by the fact that, in a moment's time, I will have moved according to some (e.g. position in the Milky Way), but I may not have moved according to others (e.g. position on the surface of the earth). (ii) It could also be argued that a tokening of the word "here" only has a definite content provided that I am not moving too quickly or too erratically. It takes time to produce a tokening of a word -- and during even a very small time interval it is possible that I may have moved a very great distance. (iii) On some views of the mind, it may be possible for me to have no spatial location. In particular, certain versions of substance dualism may have this consequence. But do I know a priori that these theories are false? (Do I know a priori that I am not an electron? Perhaps the modal interpretation of quantum mechanics is true, so that, qua electron, I quite literally have no location for much of the time.) 
In the earlier discussion, I also took for granted the assumption that any tokening of the word "now" will have a definite content (the time of the context of utterance). But there are also reasons for being sceptical about this assumption. In particular, I doubt that it is correct to claim that we know a priori that time travel is impossible. (In particular, recall Godel's claim -- supported by Einstein -- that time travel would be permitted by General Relativity if only one could get sufficient fuel into one's spacetimecraft.) But now note, as before, that any utterance of the word "now" will take time -- during which one might have been rapidly transported through a number of different times. In these circumstances, it seems that it would be wrong to say that one has any grasp -- let alone an $a$ priori grasp -- of the time of one's utterance. (I think that one may also be able to parallel the first and the third arguments which I gave for the spatial case in the temporal case; however, these arguments would be more difficult, and I shan't attempt to give them here.)

Finally, in the earlier discussion, I took for granted the assumption that any tokening of the word "I" will have a definite content (the agent of the context of utterance). But there may even be reasons for being sceptical about this assumption. In particular, consider the following sceptical hypothesis: There is a demon who has constructed a world in which there is a series of momentarily existent subjects, each of which in involved in the thinking of a single (apparent) thought. (Perhaps we don't want to call the series of "bits" of thought a single thought. Nonetheless, it is not obvious that we should deny that this hypothesis is intelligible. Think of the way in which a cartoon creates the impression of motion out of a series of still drawings. Why couldn't the illusion of the possession of a thought by a single subsisting subject be created by the stringing together of very thin temporal slices of the thoughts of distinct (but internally phenomenologically identical) subjects? If this scenario is intelligible, then it will be possible for an utterance of "I" to fail to have a definite content.

${ }^{31}$ Salmon attempts to respond to the above line of argument in "How Not To Become A Millian Heir", at pp.171-172. It seems to me that what he says that entails that he should hold either (i) that, 'in the relevant sense", there are no contingent propositions which can be known a priori; or else (ii) that, "in the relevant sense", every true contingent proposition can be known a priori (at least given the appropriate existential qualifications -- cf. footnote 4 above).

32See my “A Semantics For Propositional Attitude Ascriptions” Philosophical Studies, June 1992, for details. Notice, in particular, that, on this theory, de dicto belief ascriptions exhibit context-sensitivity and interest-relativity. Salmon strongly suggests -- in "How To Measure The Standard Metre", p.214n19 -- that he would reject any theory with this consequence. It would be interesting to know what sorts of arguments might be given in favour of his position.

${ }^{33}$ Once again, I omit the appropriate existential qualifications. The inclusion of the "strictly speaking" qualifications may be unnecessary, depending on the assumptions which one makes about the context of my work. (Cf. the discussion in section II above.)

${ }^{34}$ Of course, there is still room for a third line -- viz. that, while there are "indexical" ways of taking objects which are naturally associated with the indexicals "here", "now" and "I", there are no "indexical" ways of taking objects which can be associated with Kaplan's "dthat"-operator. Perhaps an account of "indexical" ways of taking could make this view plausible -- but I think that, at least prima facie, this is most unlikely.

${ }^{35}$ Here is one final reason for preferring the neo-Fregean theory to the one which Salmon defends: On two separate occasions, I overhear conversations in which people are talking about "Julius". From contextual clues -- perhaps even as a result of being told -- I learn that the participants in the conversation take their use of the name to be denoting (i.e. they mean to be making literal and true assertions). In the one case, the name is "descriptional" (in Evans' sense); in the other case, the name is "Millian" (in Kripke's sense). In each case, it seems to me that I can infer that Julius is Julius -- and, hence, that I can come to believe that Julius is Julius. However, according to Salmon, I am mistaken: strictly speaking, I can only come to believe that Julius is Julius in the second case. In the first case, all that I come to believe, strictly speaking, is that the sentence "Julius is Julius" expresses a true proposition. 
This seems counter-intuitive enough. But now consider the following: We can stipulate that, when the "descriptional" name "Julius" is introduced, the people who introduce it do so while pointing to a zip ("Julius" is the person who invented this kind of thing). Indeed, we can even stipulate that, when the "descriptional" name "Julius" is introduced, the people who introduce it do so while pointing to the original zip -- perhaps they are in a museum in which there is a (true) sign which says: "the original zip" -- ("Julius is the person who invented this very thing"). Even in these cases, as I read him, Salmon would say that one does not thereby come to know that Julius is Julius. (After all, if one said that, it is hard to see how one could deny that one knows a priori that Julius invented the zip! For what we seem to have is a way of thinking of Julius in which we think of him as the person who invented the zip.)

On the other hand, as I read him, Salmon holds that, e.g., the discovery of the planet Neptune -- at the time at which Leverrier fixed the reference of the planet by use of (something like) the description "the planet which causes such-and-such perturbations in the orbit of the planet Uranus -- did yield knowledge of the proposition that Neptune is Neptune (and, moreover, it also yielded a posteriori knowledge of the proposition that Neptune is the planet which causes such-and-such perturbations in the orbit of the planet Uranus). But this judgement is surely incompatible with the judgements about "Julius".

Now, perhaps I have got Salmon wrong. But there are only two alternatives: (i) Salmon can deny that Leverrier knew that Neptune is Neptune. But, in that case, it is hard to see how to decide when a claim of the form "X knows that $\mathrm{a}=\mathrm{a}$ " is, strictly speaking, true. Moreover, it is quite unclear why we should bother to speak a language which works that way. Extreme latitudinarianism makes pragmatic sense: any other convention would be arbitrary, and practically unwieldy. (Salmon claims -- "How To Measure The Standard Metre", p.199n8 -- that Kripke has an example which refutes "extreme latitudinarianism". It would be nice to see the example.) (ii) Salmon can accept that the "descriptional" reference-fixer knows that Julius is Julius. But, in that case, it seems that one will be obliged to accept that there are descriptional ways of taking objects -- and hence, that one will be obliged to accept that there are many contingent propositions which can be known a priori.

One issue which needs to be investigated here is the role of experience in the background beliefs which support reference-fixing acts. Suppose for example, that I stipulate that "Oldman1" is to name the last person born in Ballarat during the nineteenth century. And suppose further, that I have introduced a very long list of tie-breaking considerations in case that first stipulation is not sufficient to uniquely pick out a person. Given a sufficiently long list of tie-breaking considerations, I can be quite certain that there is a unique person who is denoted by the name "Oldman1". But, then, why am I not able to have singular thoughts about that person? Surely I do know that Oldman1 is Oldman1.

Another issue which needs to be investigated concerns the connections which must obtain between a subject and a domain of objects before the subject can be said to have quantificational thoughts about the domain of objects. Salmon claims that the universal quantifier is used to express the property of being the universal domain of objects. See Frege's Puzzle, p.145. (Hence, I assume that restricted universal quantifiers are used to express the property of being such-and-such restricted domain of objects.) But can I really know which property that is if I am not related "de re" to all of the objects in the domain? Surely not! And yet, in that case, it seems that none of us really knows any of the universal propositions which are expressed by sentences involving unrestricted universal quantifiers! (N.B. The sense of "de re" relation intended here is collective rather than distributive. What matters is that one is suitably related to the domain of objects over which one quantifies.)

Note how these last concerns relate to the issues about sentences of the form $a=a$. Ordinarily, one would suppose that, from the proposition which is expressed by the sentence $(\forall x)(x=x)$, one can infer the proposition that $\mathrm{a}=\mathrm{a}$. However, on Salmon's view, one cannot know the proposition that $\mathrm{a}=\mathrm{a}$ unless one is de re related to the object a (even if one knows that the name $a$ denotes). This suggests -- to me, anyway -- that, really, one cannot know the proposition that $(\forall \mathrm{x})(\mathrm{x}=\mathrm{x})$ unless one is de re related to all of the objects in the domain of quantification. And yet that is surely absurd. (Perhaps Salmon could reply that, primarily, inference has to do with sentences; i.e., that there is only a derivative sense in which inference applies to propositions. But that seems wrong. Even if logical properties are primarily properties of sentences, surely any theory which introduces propositions as epistemological objects will want inference to be an operation which involves those objects. If Salmon's theory were to 
entail that inference is not the processing of cognitive information, then that would be a serious defect in the theory.)

In sum: There is a lot which needs to be sorted out here. However, I think that the considerations raised in this footnote provide at least prima facie support for the view which I (tentatively) defend in the text.

36Described in detail in my "A Semantics For Propositional Attitude Ascriptions”.

${ }^{37}$ Strictly, this overstates Salmon's position: for he is prepared to concede that there may be some kinds of descriptional ways of taking -- though not those which I have countenanced in this paper.

${ }^{38}$ Scepticism about the problematic locutions mentioned in this paragraph has been voiced by others. See, in particular, Dennett, D. (1982) "Beyond Belief" in A. Woodfield (ed) Thought And Object, Oxford University Press: Oxford and Boer, S and Lycan, W. (1986) Knowing Who, MIT Press: Cambridge MA.

${ }^{39}$ Nathan Salmon (1993) “Relative And Absolute Apriority” Philosophical Studies 69 pp.83-100.

40“Relative And Absolute Apriority”, pp.99-100n27.

${ }^{41}$ If all instances of the schema " $\mathrm{X}$ " refers to $\mathrm{X}$ are to express facts of pure semantics, then there had better be no genuinely non-referring terms -- c.f. section I, point 7 above. This would seem to be a relatively harmless concession.

${ }^{42}$ This paper is based on sections of my unpublished doctoral dissertation Attitude Problems: The Semantics Of Propositional Attitude Ascriptions, Princeton, 1990. I would like to thank Gil Harman, Scott Soames, Bas van Fraassen, Allen Hazen, Len Goddard, Steve Rieber, Richard Holton, Kai-Yee Wong, and especially David Lewis for their comments, criticisms, and advice on various earlier versions of the ideas defended in this paper, and in my dissertation in general. A version of this paper was read at the Metaphysics Conference at ANU in November 1992; I would like to thank all those participants who provided comments and suggestions. Finally, I would also like to thank Nathan Salmon for his careful and generous comments on earlier versions of this paper. 